\title{
MODELAJE DE PROTEÍNAS DE Mycobacterium tuberculosis RELACIONADAS A SU DEFENSA FRENTE AL ESTRÉS OXIDATIVO
}

\author{
CHRISTIAN SOLIS CALERO'
}

\begin{abstract}
RESUMEN
En el presente trabajo se describ̨e el uso de procedimientos bioinformáticos e información genómica de acceso libre por Internet, para identificar y caracterizar proteínas que participan en la defensa de Mycobacterium tuberculosis (MT) frente al estrés oxidativo, con el propósito de obtener nuevos blancos farmacológicos en este patógeno. Las secuencias de aminoácidos de las proteínas correspondientes a los genes $k a t G, \operatorname{ahpC}, \operatorname{ahpD}, \operatorname{sodA}, \operatorname{sod} C$ y $m g t C$ fucron comparadas con el genoma de Mycobacterium tuberculosis H37Rv a través del servidor del National Center for Biotechnology Information, determinando su posible redundancia en el genoma. Paralelamente, se obtuvo información sobre sus residuos más conservados evolutivamente por alineamiento múltiple global usando el programa MAXHOM, así como modelos de sus estructuras tridimensionales usando los métodos de «modelaje molecular comparativo" a través del servidor SWISS-MODEL o modelaje por el método de "reconocimiento de plegamiento" (threading) a través del metaservidor GENESILICO. En base al análisis genómico se puede concluir que las proteínas codificadas por los genes $k a t G$, ahpD y $m g t C$ son buenos candidatos como blancos farmacológicos debido a que no tienen similaridad significativa con alguna proteína codificada por genes presentes en el genoma humano y no presentan redundancia en el genoma del Mycobacterium tuberculosis.
\end{abstract}

Palabras clave: Mycobacterium tuberculosis, estrés oxidativo, bioinformática, genoma, modelaje molecular

\section{SUMMARY}

This work uses bioinformatics tools and free online access to genomic information in order to identify and characterize proteins related to oxidative stress defense of Mycobacterium tuberculosis, in order to discover new appropriate pharmacological targets against this pathogen. Sequences of amino acids of proteins expressed with that function, products of the expression of the genes $k a t G, \operatorname{sod} A, \operatorname{sod} C, \operatorname{ahp} C$, ahpD and $m g t C$ were compared with the genome of Mycobacterium tuberculosis H37Rv through the NCBI server National for Center Biotechnology Information), determining their possible redundancy in the genome. Parallely, information was obtained above of their evolutive conserved sequences by global multiply alignment using MAXHOM program, as well models of their threedimensional structures by comparative modeling method through the Swiss-model server, or using "recognition of folding» method (threading) through the GENESILICO metaserver. By genomic analysis, proteins codified by kat $G$, $a h p D$ and $m g t C$ genes would be very good candidates to be pharmacological targets due to absence of redundancy in the genome of Mycobacterium tuberculosis as well as similarity with any codified protein by human genes.

Key words: Mycobacterium tuberculosis, oxidative stress, bioinformatics, genome, molecular modeling

\section{INTRODUCCIÓN}

Para el diseño de nuevos fármacos, una de las nuevas estrategias es el llamado diseño racional, que comporta un conocimiento previo de la diana sobre la que se quiere actuar. La información sobre las dianas y blancos farmacológicos se encuentra de modo primario residente en el genoma, por ello una de las claves en esta estrategia es traducir esta información genómica usando varias herramientas, entre las que destaca la Bioinformática,

I Facultad de Farmacia y Bioquímica - Universidad Nacional Mayor de San Marcos. que permitan la identificación de dianas concretas y el posterior desarrollo de candidatos a fármacos. En el caso de los patógenos, las enzimas que catalizan los pasos limitantes de ciertas reacciones exclusivas de procariotas, son dianas adecuadas, fáciles de "extraer" una vez conocida la secuencia genómica. Seleccionar estas dianas resulta todo un reto para las empresas a fin de llevar a cabo esta investigación de una forma rápida y sin necesidad de mucha inversión.

Durante el proceso de infección por Mycobacterium tuberculosis (MT) en el huésped, los macrófagos generan especies químicas reactivas de oxígeno y 
nitrógeno que restringen el desarrollo del patógeno (1), el cual, para contrarrestar este efecto, ha desarrollado varios mecanismos de defensa. Dentro de las especies químicas generadas por los macrófagos, destacan los intermedios reactivos de Nitrógeno (RNI) que incluyen $\mathrm{NO}, \mathrm{NO}_{2}, \mathrm{y} \mathrm{NO}_{3^{\prime}}$ porque se ha demostrado que juegan un rol importante en controlar el crecimiento del MT en el huésped $(2,3)$. La habilidad de MT para sobrevivir al ataque de los RNI involucra un grupo de proteínas, las que por su importancia para la supervivencia de la bacteria son blancos potenciales para nuevos fármacos a desarrollar para eliminar a este patógeno del organismo, de allí el interés en este trabajo de caracterizarlas y validarlas como blancos farmacológicos usando las herramientas bioinformáticas disponibles de acceso libre en Internet.

\section{MATERIAL Y MÉTODOS}

Búsquedas en bases de datos de secuencias. De la base de datos NR (no redundante) GenBank del NCBI (National Center for Biotechnology Information) localizado en la siguiente dirección (http: / / www.ncbi.nlm.nih.gov/) utilizando el programa ENTREZ, se obtuvo registros de secuencias de aminoácidos en formato FASTA, correspondientes a proteínas relacionadas a la defensa de MT frente al estrés oxidativo (Tabla 1).

Análisis genómico. Las secuencias de aminoácidos de las proteínas seleccionadas fueron comparadas con los genomas de MT y Homo sapiens usando los programas TBLASTN y contra la lista de proteínas referenciadas de ambos organismos usando el programa BLASTP a través de los servidores del NCBI, para determinar su presencia, localización, así como la posible redundancia de sus correspondientes genes en estos genomas. La información sobre el papel de las proteínas en el metabolismo de MT fue obtenida en el Tuberculist Web Server (Instituto Pasteur: http:// genolist. pasteur.fr/TubercuList/ ).

Caracterización de las secuencias de aminoácidos. A través del servidor PREDICT PROTEIN http://cubic.bioc.columbia.edu/ predictprotein, se calculó:

- Estructura secundaria de las proteínas usando el programa PROFSEC

- Búsqueda de motivos usando el programa PROSITE

- Alineamiento múltiple global usando el programa MAXHOM.

- Predicción del \% de residuos de aminoácidos expuestos (en más del 16\% de su superficie), midiendo la accesibilidad del solvente con el programa PROFSEC.
Predicción de las estructuras tridimensionales. Previamente se realizó una búsqueda por similaridad en la base de datos de estructuras 3D del NCBI usando el programa BLASTP. De presentar la secuencia problema una similaridad significativa con la secuencia de alguna proteína de estructura 3D conocida se realizó la aproximación a sus estructuras tridimensionales por modelaje molecular comparativo a través del servidor SWISS-MODEL (http://www.expasy.ch), en su defecto la aproximación se realizó por el método de "reconocimiento de plegamiento" ("threading") a través del metaservidor para predicción de plegamiento GENESILICO (http://genesilico.pl/ meta). La calidad de las estructuras tridimensionales fue evaluada usando el programa VADAR 1.4 (http://redpoll.pharmacy. ualberta.ca/vadar). El análisis de los modelos de las estructuras tridimensionales obtenidas en busca de los residuos de aminoácidos más conservados, se realizó usando el visor de estructuras 3D RASMOL y la información obtenida por el alineamiento múltiple global obtenido con el programa MAXHOM usando varias secuencias similares de otras especies.

\section{RESULTADOS Y DISCUSIÓN}

Al analizar el genoma de MT con el fin de desarrollar nuevos fármacos, el procedimiento a seguir pasa primero por el uso de herramientas bioinformáticas para identificar y caracterizar los genes de interés adicionalmente, usando dicha información se puede dar la utilización de técnicas de análisis de la expresión diferencial de genes (DNA microarrays), acopladas con la disciplina emergente de la química combinatoria. Un aspecto clave en la infección causada por MT es su supervivencia en el interior de los macrófagos, ya que estas células son capaces de destruir más del $90 \%$ de los bacilos (4). Para sobrevivir MT debe evitar los mecanismos bactericidas de estas células, una de las estrategias es a través de la detoxificación de los reactivos intermediarios del oxígeno o del nitrógeno, es decir defenderse del estrés oxidativo al que es sometido por el huésped.

En la tabla 1 se presenta una lista de las proteínas de MT seleccionadas para este estudio que participan en la defensa frente al estrés oxidativo, entre ellas destaca el sistema Catalasa-peroxidasa codificada por el gen $K a t G$ que defiende al patógeno de las especies reactivas de oxígeno $(1,5,6)$. Esta catalasa-peróxidasa, es responsable de la conversión de la Isoniazida a su forma biológicamente activa contra el patógeno (7). Las mutaciones en katG que codifican para una catalasa/ peroxidasa incapaz de convertir la isoniacida a su forma acti- 
va, confieren resistencia a este fármaco, entonces queda como protector antioxidante el sistema alkyl hidroperóxido reductasa $(A h p C / A h p D)$ que es capaz de detoxificar peróxidos orgánicos y, probablemente, también peróxido de hidrógeno, su actividad se atribuye a la presencia en su estructura de un par de residuos de cisteina (Cys) (8). Dos genes en MT codifican las enzimas superóxido dismutasas, $\operatorname{Sod} A$ [Fe] y SodC [Cu-Zn]. Estas proteínas parecen jugar un papel importante protegiendo a la bacteria de la acción de los intermediarios reactivos del oxígeno. SodA a diferencia de SodC, es una proteína dependiente de la proteína SecA2 para su secreción (9). Bacterias mutantes de los genes que codifican estas proteínas han mostrado ser susceptibles a los mecanismos bactericidas de macrófagos peritoneales activados con interferón gamma (INF-ã) usando ratón como modelo experimental (10). Otra proteína incluida en este estudio es una proteína $C$ transportadora de magnesio codificada por el gen $m g t C$, la disrupción de este gen provoca un crecimiento alterado del microorganismo en macrófagos derivados de monocitos humanos y menor supervivencia en ratones $\mathrm{BALB} / \mathrm{c}$ infectados por vía venosa, lo cual indica que el gen $m g t C$ juega un papel importante en el crecimiento de MT en macrófagos humanos y en su virulencia en ratones (11). El transporte de cationes como el $\mathrm{Mg}^{2+}$ parece ser uno de los mecanismos que le permite a MT sobrevivir dentro de un fagosoma ligeramente acídico y limitado en $\mathrm{Mg} 2+$ (12).

TABLA 1. CARACTERÍSTICAS BIOQUÍMICAS DE LAS PROTEÍNAS ESTUDIADAS

\begin{tabular}{|c|c|c|c|c|c|}
\hline GEN & Proteína & $\begin{array}{l}\text { Número de } \\
\text { aminoácidos }\end{array}$ & $\begin{array}{c}\text { Peso } \\
\text { molecular } \\
\text { (Daltons) }\end{array}$ & $\begin{array}{c}\text { Punto } \\
\text { Isoeléctrico }\end{array}$ & $\begin{array}{c}\text { Acceso a la } \\
\text { secuencia } \\
\text { (ENTREZ) }\end{array}$ \\
\hline katG & $\begin{array}{l}\text { Sistema Catalasa- } \\
\text { peroxidasa }\end{array}$ & 740 & 80600.37 & 4.8700 & NP_216424.1 \\
\hline sodA & $\begin{array}{l}\text { Superóxido } \\
\text { dismutasa A }\end{array}$ & 207 & 23032.65 & 6.4323 & NP_218363.1 \\
\hline sodC & $\begin{array}{l}\text { Superóxido } \\
\text { dismutasa C }\end{array}$ & 240 & 23843.13 & 6.4255 & NP_214946.1 \\
\hline ahpC & $\begin{array}{l}\text { Sistema alquil } \\
\text { hidroperoxido }\end{array}$ & 195 & 21565.05 & 4.2810 & NP_216944.1 \\
\hline ahpD & reductasa & 177 & 18779.40 & 7.0572 & NP_216945.1 \\
\hline mgtC & $\begin{array}{c}\text { Proteína } \mathrm{C} \\
\text { transportadora de } \\
\mathrm{Mg}\end{array}$ & 234 & 24805.22 & 8.4887 & NP_216327.1 \\
\hline
\end{tabular}

En la tabla 2 se presenta el análisis genómico, que permite validar las proteínas en estudio como posibles blancos farmacológicos. En este análisis se evalúa la redundancia, que puede presentarse cuando para una proteína hay en el genoma más de un segmento con similaridad, lo que puede ser una señal que para la actividad de esa proteína existen isoformas codificadas por distintos genes bastante similares pero no iguales, lo que dificultaría el hallar un inhibidor para su actividad, ya que los sitios de unión al ligando no serian exactamente los mismos presentándose diferentes valores de afinidad y de actividad intrínseca. De acuerdo a los resultados obtenidos de las 6 proteínas analizadas, todas, excepto la correspondiente al gen $\operatorname{ahpC}$, no presentan una señal de redundancia (Figura 1). Los criterios para identificar los segmentos son los valores de $\mathrm{E}$ (probabilidad de que el alineamiento obtenido sea producto del azar) que deben ser superiores a $10^{-6}$, los valores de Score (una medida de la significancía estadística) que deben ser superiores a 50 bits, y la longitud del alineamiento (se muestra en la columna posición del alineamiento en la proteína de la tabla 2), el análisis de este último parámetro es importante porque frecuentemente alineamientos muy cortos presentan valores $\mathrm{E}$ altos, lo que no es confiable. Se comparó también las secuencias de las proteínas frente a la información de secuencia del genoma humano. Es un criterio de validación que las proteínas del MT, que presenten una similaridad 
significativa con alguna proteína humana, deben ser descartadas como posibles blancos farmacológicos ya que es probable que los antagonistas de es- tas proteínas presenten también algún grado de unión específica a proteínas codificadas por genes del genoma humano, lo que, a la larga, se traduci-

TABLA 2. EVALUACIÓN DE LAS PROTEÍNAS SELECCIONADAS EN BASE A DATOS GENÓMICOS

\begin{tabular}{|c|c|c|c|c|c|c|c|}
\hline \multirow[t]{2}{*}{ PROTEÍNA } & \multicolumn{3}{|c|}{ Segmentos similares } & \multicolumn{2}{|c|}{$\begin{array}{c}\text { Parámetros } \\
\text { (BLASTP) } \\
\text { Genoma MT } \\
\text { H37TRV } \\
\end{array}$} & \multicolumn{2}{|c|}{$\begin{array}{c}\text { Parámetros } \\
\text { (BLASTP) } \\
\text { Genoma } \\
\text { Humano } \\
\end{array}$} \\
\hline & $\begin{array}{l}\text { Gen } \\
\text { similar }\end{array}$ & $\begin{array}{l}\text { Posición del } \\
\text { alineamiento } \\
\text { en la proteína } \\
\text { (aminoácidos) }\end{array}$ & $\begin{array}{l}\text { Posición del gen } \\
\text { el genoma de MT } \\
\text { (nucleótidos) }\end{array}$ & Score & E value & Score & E value \\
\hline $\begin{array}{l}\text { Sistema Catalasa - } \\
\text { peroxidasa KatG }\end{array}$ & $k a t G$ & $1-740$ & $2153889-2156111$ & 1494 & 0.0 & \multicolumn{2}{|c|}{$\begin{array}{c}\text { No se encontró } \\
\text { similaridad }\end{array}$} \\
\hline $\begin{array}{l}\text { Superóxido } \\
\text { dismutasa A sodA }\end{array}$ & $\operatorname{sod} A$ & $1-207$ & $4320704-4321327$ & 434 & $e-123$ & 207 & $3 e-54$ \\
\hline $\begin{array}{l}\text { Superóxido } \\
\text { dismutasa } C \text { sodC }\end{array}$ & $\operatorname{sod} C$ & $1-240$ & $519600-520322$ & 451 & e- 128 & 50 & $2 \mathrm{e}-06$ \\
\hline \multirow{4}{*}{$\begin{array}{l}\text { ahpC: alkyl } \\
\text { hidroperóxido } \\
\text { reductasa }\end{array}$} & $\operatorname{ahpC}$ & $1-195$ & $2726193-2726780$ & 399 & e-113 & \multirow[b]{4}{*}{122} & \multirow[b]{4}{*}{$1 e-28$} \\
\hline & $a h p E$ & $49-146$ & $2510715-2511176$ & 52 & $2 \mathrm{e}-08$ & & \\
\hline & $b c p B$ & $49-170$ & $1807298-1807762$ & 48 & $4 \mathrm{e}-07$ & & \\
\hline & $b c p$ & 4-144 & $2837684-2838157$ & 44 & $7 e-06$ & & \\
\hline $\begin{array}{l}\text { ahpD: alkyl } \\
\text { hidroperóxido } \\
\text { reductasa }\end{array}$ & $a h p D$ & $1-177$ & $2726806-2727339$ & 322 & $9 e-90$ & \multicolumn{2}{|c|}{$\begin{array}{c}\text { No se encontró } \\
\text { similaridad }\end{array}$} \\
\hline $\begin{array}{l}\text { Proteína } \mathrm{C} \\
\text { transportadora de } \\
\mathrm{Mg}(\mathrm{mgtC})\end{array}$ & $m g t C$ & $1-234$ & $2053443-2054147$ & 462 & $e-131$ & \multicolumn{2}{|c|}{$\begin{array}{c}\text { No se encontró } \\
\text { similaridad }\end{array}$} \\
\hline
\end{tabular}

ría en efectos adversos para el paciente de usar esos antagonistas como fármacos contra el MT. Los resultados de este estudio (tabla 2) muestran que

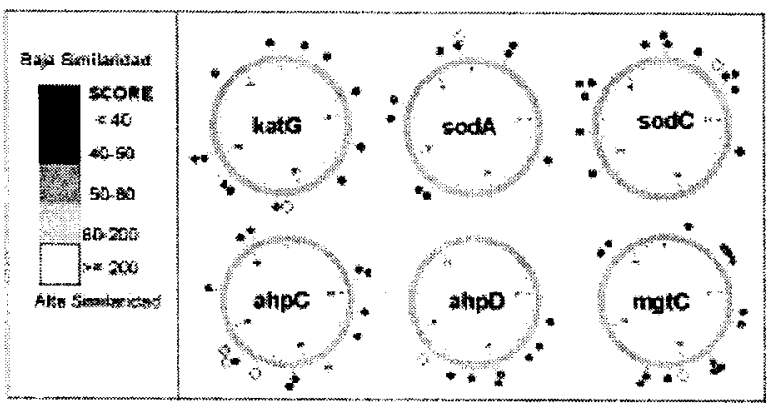

Figura 1. Análisis genómico de la redundancia en el genoma del MT para las proteínas estudiadas. Cada punto alrededor del genoma representado como un círculo señala la similaridad encontrada y la escala de grises la significancía de ésta. las proteínas codificadas por los genes $k a t G, a h p D$, $m g t C$ no presentan ninguna similaridad significativa con proteína alguna de origen humano.

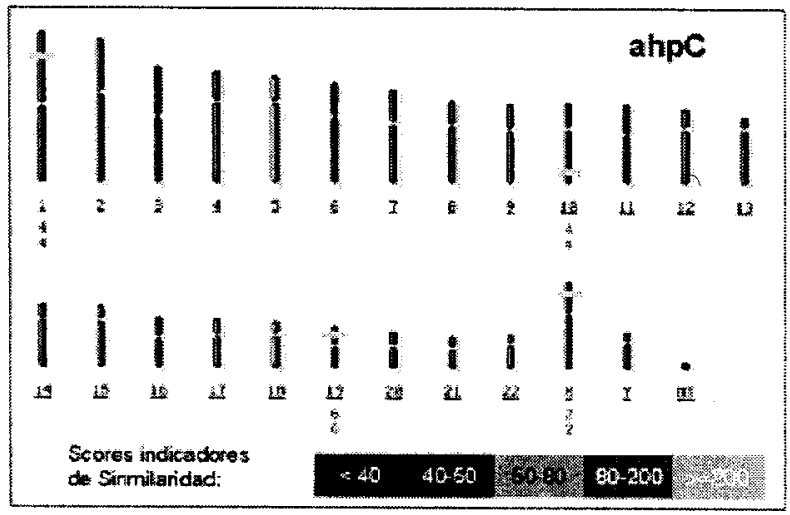

Figura 2. Similaridad de la proteína codificada por el gen ahpC de Mycobacterium tuberculosis a proteínas codificadas por genes del genoma humano 
La tabla 3 muestra los datos obtenidos en los cálculos de predicción de la estructura secundaria de las proteínas en estudio. Se señala además los métodos que se han usado para obtener información de sus estructuras tridimensionales, así como la evaluación de la calidad de estos modelos a través del programa VADAR 1.4 (13). La información sobre la estructura tridimensional obtenida de modo experimental para las proteínas codificadas por los genes katG, ahpD, SodA y SodC, la ob- tuvimos de la base de datos PDB («Protein Data Bank»).

Las otras proteínas no presentan estructuras determinadas experimentalmente por lo que se obtuvo modelos de sus estructuras tridimensionales por los métodos de predicción de «modelado por homología" en el caso de los productos del gen $a h p C$, y el método de «reconocimiento de plegamiento» (threading) que se aplicó en el caso del

TABLA 3. CARACTERÍSTICAS DE LAS ESTRUCTURAS 3D DE LAS PROTEÍNAS SELECCIONADAS

\begin{tabular}{|c|c|c|c|c|c|}
\hline \multirow[t]{2}{*}{ Proteína } & \multicolumn{3}{|c|}{$\begin{array}{c}\text { \% de Estructuras } \\
\text { secundarias (PROFsec) }\end{array}$} & \multirow{2}{*}{$\begin{array}{l}\text { Método de obtención } \\
\text { de la Estructura } \\
\text { tridimensional }\end{array}$} & \multirow{2}{*}{$\begin{array}{c}\text { Calidad de la } \\
\text { estructura } \\
\text { (Vadar 1.4) }\end{array}$} \\
\hline & $\alpha$ hélices & Hojas- $\beta$ & lazos & & \\
\hline $\begin{array}{l}\text { Sistema Catalasa- } \\
\text { peroxidasa (KatG) }\end{array}$ & 32.43 & 8.51 & 59.05 & $\begin{array}{l}\text { Difracción por rayos X } \\
\text { (Código PDB:1SJ2) }\end{array}$ & $5 / 10$ \\
\hline $\begin{array}{l}\text { Superóxido } \\
\text { dismutasa A sodA }\end{array}$ & 53.62 & 9.18 & 37.20 & $\begin{array}{l}\text { Difracción por rayos X } \\
\text { (Código PDB: } \underline{\text { IDS })}\end{array}$ & $7 / 10$ \\
\hline $\begin{array}{l}\text { Superóxido } \\
\text { dismutasa } C \text { sodC }\end{array}$ & 12.50 & 25.42 & 62.08 & $\begin{array}{l}\text { Difracción por rayos X } \\
\text { (Código PDB: } \underline{1 \mathrm{PZS}} \text { ) }\end{array}$ & $7 / 10$ \\
\hline $\begin{array}{l}\text { Sistema alkyl } \\
\text { hidroperóxido } \\
\text { reductasa (ahpC) }\end{array}$ & 19.49 & 18.97 & 61.54 & $\begin{array}{l}\text { Modelaje comparativo } \\
\text { (SWISS MODEL) }\end{array}$ & $6 / 10$ \\
\hline $\begin{array}{l}\text { Sistema alkyl } \\
\text { hidroperóxido } \\
\text { reductasa (ahpD) }\end{array}$ & 80.23 & 0 & 19.77 & $\begin{array}{l}\text { Difracción por rayos X } \\
\text { (Código PDB: } \underline{1 \mathrm{GU} 9} \text { ) }\end{array}$ & $7 / 10$ \\
\hline $\begin{array}{l}\text { Proteína } C \\
\text { transportadora de } \\
\text { magnesio (mgtC) }\end{array}$ & 48.72 & 19.23 & 32.05 & $\begin{array}{c}\text { Threading } \\
\text { (MGENTHREADER }\end{array}$ & $6 / 10$ \\
\hline
\end{tabular}

gen $m g t C$, usando el Metaservidor GeneSilico. En este último caso, se seleccionó el modelo generado por el programa MGenTHREADER, por presentar los valores más confiables respecto a otros métodos de threading como 3dpssm, Bioinbgu, Ffas03, Fugue 2,0,Pcons, Sam-T02 y sparks.

Estos dos tipos de métodos usan información que proviene, básicamente, de otras proteínas cuya estructura se ha determinado experimentalmente. Los métodos de "modelado por homología» se basan en la similaridad que presenta la secuencia de aminoácidos de la proteína de interés con las secuencias de proteinas cuya estructura tridimensional ya se ha determinado experimentalmente (14). Cuando la secuencia de aminoácidos de la proteína analizada presenta baja similaridad con proteínas cuya estructura 3D se conoce, no se puede aplicar el modelado por homología, por lo que se uso el método "threading". En este método se busca que estructuras de conocida estructura 3D, presenten el mejor modelo de plegamiento al que corresponda la secuencia problema (15). La estrategia general de estos programas, como el MGenTHREADER, consiste en evaluar la secuencia problema en una serie de plegamientos y encontrar en cual de ellos esta secuencia se "encaja» o «se encuentra bien». MGenTHREADER combina varios métodos incluyendo alineamiento, potenciales de solvatación y potenciales entre pares, finalmente evalúa el alineamiento usando una red neuronal (16). Con la Proteína $C$ transportadora de $\mathrm{Mg}$, (mgtC) sólo se obtuvo un modelo con valores confiables usando MGenTHREADER, pero que no pudo validarse con otros métodos ni de modelaje por homología y threading por lo que se constituye en un objeto de estudio para el afinamiento del modelo por métodos propios de la Química Computacional usando la mecánica molecular o cuántica, métodos que involucran un 
mayor costo computacional en tiempo, pero debido al bajo peso molecular de esta proteína ( 234 aminoácidos) respecto a las otras proteínas en estudio, ello se puede hacer factible sobre todo para zonas de la proteína en los que no hay ninguna semejanza a los plegamientos conocidos.

Los sitios de unión a ligandos, así como los sitios activos, presentan generalmente residuos que han sido conservados en la evolución, debido a su importancia para la función de la proteína, por ello es posible detectarlos por alineamiento múltiple. Con ese propósito se uso el programa MAXHOM, usando los alineamientos múltiples se pudo identificar estos residuos para luego buscar su posi- ción en la estructura 3D de la proteína usando un programa de visualización de estructuras 3D como RASMOL. En la Figura 4 se presenta los resultados correspondientes a la alquil hidroperóxido reductasa codificada por el gen $a h p C$; destaca el hecho de que varios residuos altamente conservados en el alineamiento múltiple como la Asp 88 , Ser 86 , Ser 89 y la His 93 se presenten muy cerca una de la otra y las tres primeras formando parte de un «loop» o lazo proteico, estructura secundaría que muy frecuentemente aparte de conectar otros tipos de estructuras secundarias, está relacionada a los sitios activos de las enzimas (17). Se hace énfasis en estos residuos porque son caracte-

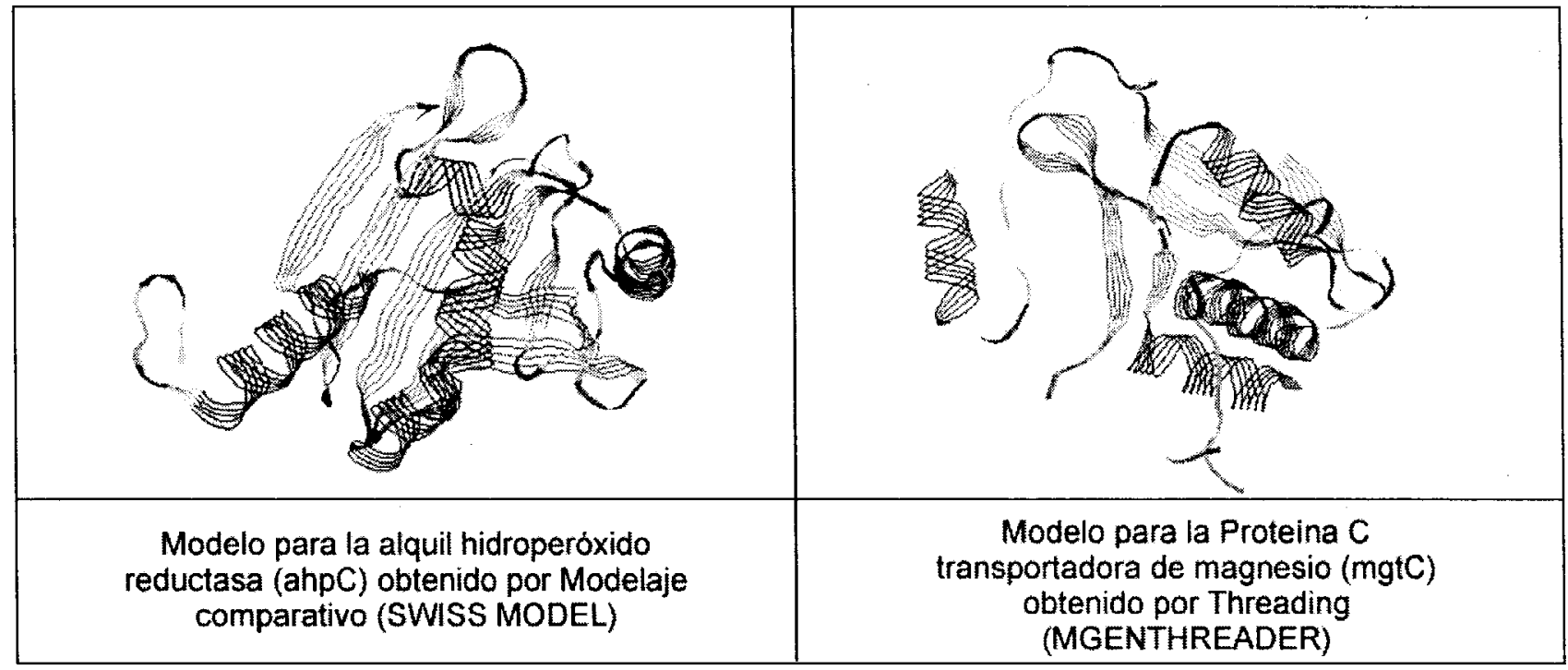

Figura 3. Modelos de la estructura tridimensional obtenidos por modelaje molecular usando secuencias de aminoácidos

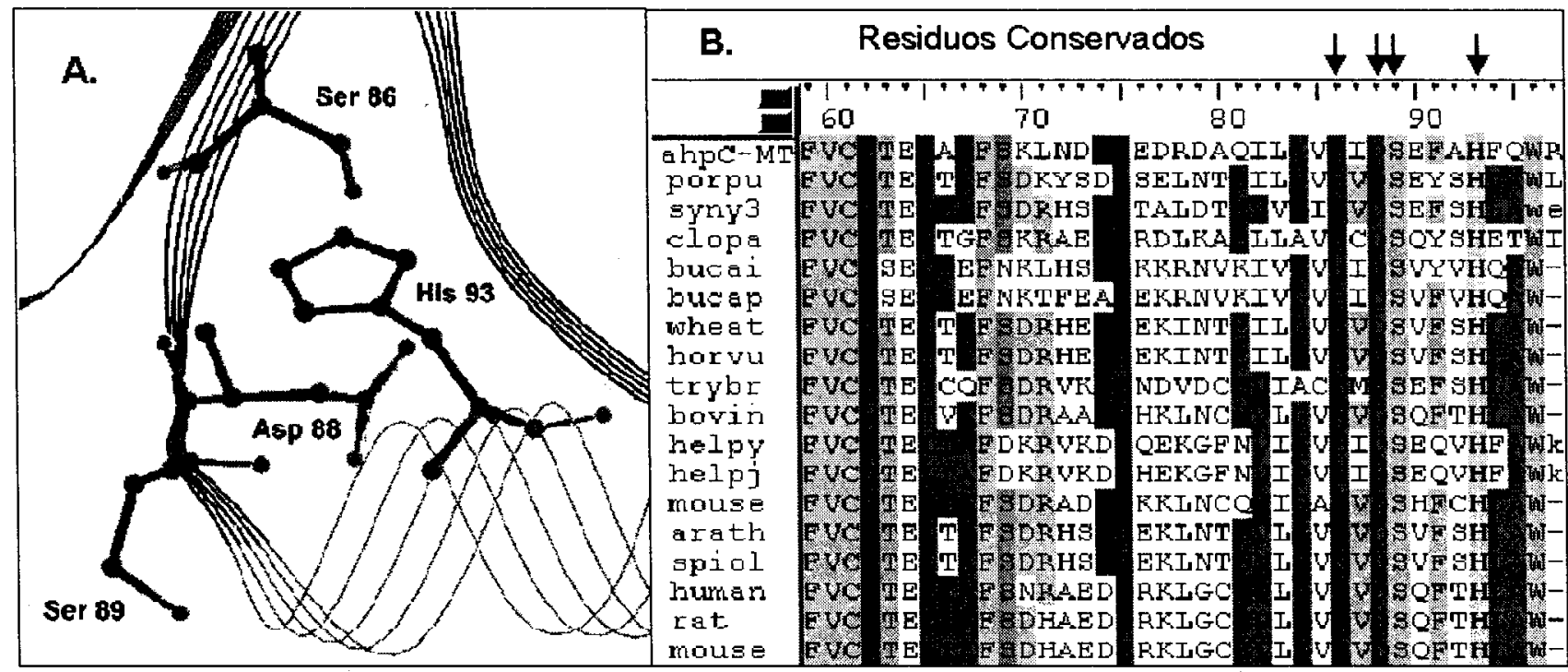

Figura 4. Análisis del Modelo de la alquil hidroperóxido reductasa codificada por el gen ahpC . A: Residuos conservados característicos de centros activos enzimáticos. B: Alineamiento múltiple obtenido con el programa MAXIIOM mostrando los residuos conservados. 
rísticos de los centros activos de las enzimas y porque los otros residuos conservados correspondientes a aminoácidos aromáticos y alifáticos que forman bloques en el alineamiento, son más bien característicos de los núcleos o «core» de las proteínas, regiones de la proteína muy importantes para la estabilidad de la estructura de la proteína aunque no están relacionados de manera directa a su rol catalítico (18).

Se concluye que las proteínas producto de los genes $k a t G, a h p D$ y $m g t C$ en base a los análisis bioinformáticos realizados, presentan las mejores características desde el punto de vista genómico para ser consideradas como blancos farmacológicos. Como se dispone en algunos casos de modelos de estructuras 3D obtenidos experimentalmente y en otros casos por Modelaje molecular, es factible usar esa información para posteriores ensayos computacionales y experimentales que permitan el diseño de nuevos ligandos antagonistas de estas proteínas, que luego de un proceso de optimización se puedan constituir en nuevos fármacos. La información obtenida por el trabajo «in silico» proporciona una aproximación complementaria que puede ser la base para el trabajo experimental «in vitro».

\section{REFERENCIAS BIBLIOGRÁFICAS}

1. Manca C, Paul S., Barry CE., Freedman V.H, Kaplan G. Mycobacterium tuberculosis catalase and peroxidase activities and resistance to oxidative killing in human monocytes in vitro. Infect. Immun 1999; 67:7479

2. Chan, J., Xing Y., Magliozzo RS. Bloom B.R. Killing of virulent Mycobacterium tuberculosis by reactive nitrogen intermediates produced by activated murine macrophages. J. Exp. Med. 1992; 175:1111-1122.

3. MacMicking JD., North RJ., Lacourse R., Mudgett JS., Shah SK. Nathan C.F. Identification of nitric oxide synthase as a protective locus against tuberculosis. Proc. Natl Acad. Sci. USA 1997; 94:5243-5248

4. Fenton MJ, Vermeulen MW. Immunopathology of tuberculosis: roles of macrophages and monocytes. Infect Immun. 1996; 64(3):683-90.

5. Heym B., Zhang Y., Poulet S., Young D. Cole S.T. Characterization of the katG gene encoding a catalase-peroxidase required for the isoniazid susceptibility of Mycobacterium tuberculosis. J. bacteriol 1993; 175:4255-4259.

6. Flynn JL. Chan J. Immunology of tuberculosis. Annu. Rev. Immunol 2001; 19:93-129.
7. Koshkin A, Nunn C, Djordjevic S, Ortiz de Montellano P. The Mechanism of Mycobacterium tuberculosis Alkylhydroperoxidase AhpD as Defined by Mutagenesis, Crystallography, and Kinetics. Journal Biol Chem 2003; 278(32):29502-08

8. Bryk R, Griffin P, Nathan C. Peroxynitrite reductase activity of bacterial peroxiredoxins. Nature 2000; 407: 211-15

9. Braunstein M, Espinosa BJ, Chan J, Belisle JT, Jacobs WR. SecA2 functions in the secretion of superoxide dismutase $A$ and in the virulence of Mycobacterium tuberculosis. Mol Microbiol 2003; 48(2):453-64.

10. Piddington DL, Fang FC, Laessig T, Cooper AM, Orme IM, Buchmeier NA. Cu, Zn superoxide dismutase of Mycobacterium tuberculosis contributes to survival in activated macrophages that are generating an oxidative burst. Infect Immun 2001; 69(8):4980-7.

11. Chacón O, Realpe T, Barletta R, Robledo J. Inactivación de genes de Mycobacterium tuberculosis y su potencial utilidad en la prevención y el control de la tuberculosis. Biomédica 2004; 24:165-87

12. Buchmeier N, Blanc-Potard A, Ehrt S, Piddington D, Riley L, Groisman EA. A parallel intraphagosomal survival strategy shared by Mycobacterium tuberculosis and Salmonella enterica. Mol Microbiol 2000; 35(6):1375-82.

13. Morris A., MacArthur M., Hutchinson, E. Stereochemical quality of protein structure coordinates. Proteins 1992; 12(4):345-364

14. Schwede T, Kopp J, Guex N, Peitsch MC. SWISS-MODEL: an automated protein homology-modeling server. Nucleic Acids Res 2003; 31:3381-3385.

15. Fischer D, Barret C., Bryson K. CAFASP-1: Critical Assessment of Fully Automated Structure Prediction Methods. Proteins: Structure, Function and Genetics 1999: 3:209217

16. Jones D. GenTHREADER: An Efficient and Reliable Protein Fold Recognition Method for Genomic Sequences. J Mol Biol 1999; 287(4):797-815.

17. Branden $C$, Tooze J. Introduction to protein structure. Garland Publishing New York 1991.

18. Claverie J-M. Notredame C. Building a Multiple Sequence Alignment. Bioinformatics for Dummies, New York, Wiley Publishing Inc., 2003; pp 279-314 